\section{In the mood for regeneration}

\section{By Tim Fulmer, Senior Writer}

Researchers at the University of Texas Southwestern Medical Center have found that lithium, the marketed mood stabilizer, might have utility in spinal cord injury, an indication for which there are no approved drugs. ${ }^{1}$ In a paper in The Journal of Neuroscience, the group showed that lithium promoted axonal growth in rat models of SCI. Other researchers next want to see more preclinical work to better elucidate the mechanism of lithium in SCI.

Injury to the spinal cord results in damaged neurons, glial cells, blood vessels and meninges. Moreover, cellular debris at the injury site contains numerous molecules that potentially inhibit regrowth of neurons. When these molecules bind to receptors on the surface of damaged neurons, they trigger an intracellular pathway-the ras homolog gene family, member A (RhoA)-Rho-associated, coiledcoil containing protein kinase (ROCK) pathway-that irreversibly inhibits neurite sprouting and regeneration (see Figure 1, "Blocking growth inhibition in spinal cord injury")., ${ }^{2,3}$

Consequently, many therapeutic strategies in SCI have sought to block this growth inhibitory pathway (see Table 1, "Spinal cord injury pipeline").

The UT Southwestern researchers discovered that inhibiting glycogen synthase kinase $3 \beta$ (GSK3 $\beta$ ), an enzyme already implicated in neuronal polarity and axonal elongation, ${ }^{4,5}$ induced neuron growth in cell culture and animal models of SCI.

Cultured dorsal root ganglion neurons treated with either of two GSK3 $\beta$ inhibitors - lithium or SB415286- had enhanced neurite outgrowth and axonal elongation in the presence of myelin and chondroitin sulfate proteoglycan (CSPG) compared with what was seen in cells that received vehicle control. Myelin and CSPG are known to inhibit neuronal growth in vivo following injury.

Lithium is a generic noncompetitive inhibitor of GSK3 $\beta$ that is marketed to treat mania associated with bipolar disorder. SB415286 is a small molecule competitive inhibitor of GSK3 $\beta$ that was originally developed by SmithKline Beecham, now part of GlaxoSmithKline plc. ${ }^{6}$ The pharma company did not return calls regarding the status of SB415286.

SB415286 is an ATP analog that competes with ATP substrate for GSK3 $\beta$ 's active site, whereas lithium displaces magnesium ions in GSK $3 \beta$ and therefore does not compete with ATP substrate for the active site.

In three rodent models of SCI, systemic delivery of lithium pro- moted regeneration of corticospinal tract axons compared with what was seen in vehicle-treated littermates. In two of the models, lithium also improved the functional endpoint of locomotion compared with that seen in rodents that received vehicle control.

\section{Easy dose it}

Shuxin $\mathrm{Li}$, corresponding author on the paper and assistant professor of neurology at UT Southwestern, told SciBX that "it is very important now to identify the optimal dose of lithium for axon growth in animal models before moving into a clinical trial." A single dose of lithium $(3 \mathrm{mEq} / \mathrm{kg})$ was tested in the paper.

The label of Eskalith lithium carbonate states that therapeutic serum levels of the compound typically fall in the $0.6-1.5 \mathrm{mEq} / \mathrm{L}$ range in bipolar patients. Li said identifying an optimal lithium dose will probably require measuring serum levels of the compound in the murine SCI models.

The good news, said William Snider of the University of North Carolina School of Medicine, is that lithium already has shown safety in bipolar disorder. Thus, it could have a smoother transition to clinical trials in SCI than newer therapeutic strategies that have shown promise in preclinical models.

Snider, director of the UNC Neuroscience Center, and colleagues are studying the role of neurotrophins in the development and regeneration of axons.

Snider also said that additional preclinical work is needed to discover the mechanism of GSK3 $\beta$ inhibitors in SCI and determine if certain neuronal cell types are affected differently than others at a given dose. The article in the Journal of Neuroscience could also prompt the investigation of other GSK3 $\beta$ inhibitors, he added (see Box 1, "Inhibiting GSK3 in cancer").

Li said his lab plans to study other GSK3 $\beta$ inhibitors.

Companies in the SCI space were optimistic about targeting GSK3 $\beta$.

Because both noncompetitive inhibitors such as lithium and competitive specific inhibitors such as SB415286 showed efficacy, GSK $3 \beta$ is likely a credible target in SCI, said Mark Pykett, president and COO of Alseres Pharmaceuticals Inc.

"Lithium's long clinical history, oral bioavailability and ability to cross the blood-brain barrier would suggest that it is an interesting candidate for further drug development in SCI, although pharmacology and toxicity issues surrounding dose and delivery would need to be ironed out," he said.

In the meantime, Pykett added, "studies in GSK3 $\beta$ knockout mice could offer additional target validity."

Alseres' Cethrin (BA-210) - a recombinant C3 exoenzyme protein antagonist of Rho guanosine triphosphatase-will enter Phase IIb testing to treat acute SCI early next year. In a Phase I/IIa trial, 5 of 13 treated patients with cervical injury had a two-grade or better improvement in the American Spinal Injury Association (ASIA) Impairment Scale (AIS). Alseres (formerly Boston Life Sciences Inc.) has an exclusive worldwide license to Cethrin from BioAxone 


\section{Therapeutics Inc.}

Anthony Caggiano, senior director of preclinical development at Acorda Therapeutics Inc., told SciBX that inhibiting GSK3 $\beta$ "is backed by a sound rationale that supports similar strategies under investigation to treat spinal cord injury."

"We know that the generally inhibitory environment of the CNS is a key barrier to axonal regeneration following injury," he said. "Many therapeutic strategies in spinal cord injury have sought to overcome this nonpermissive environment by blocking specific proteins that likely contribute to it, including myelin-associated glycoprotein, NOGO-A protein, chondroitin sulfate proteoglycan and now perhaps GSK3ß.”

Caggiano did say there are important limitations of rodent models of SCI.
"It's not straightforward extrapolating therapeutic efficacy from the mild or moderate injuries we see in rodents to the severe spinal cord injuries we often encounter in human trials," he noted. "Secondly, the great difference in scale between mouse and human makes interpreting data on axonal elongation difficult. Consequently, ideal next steps might include looking at GSK3 $\beta$ inhibition in larger animal models of spinal cord injury, such as cats or even primates."

Acorda's recombinant chondroitinase is in preclinical development for SCI. The enzyme breaks down growth-inhibitory CSPG in the environment of damaged neurons and also helps induce neuronal plasticity.

In rat models of moderate SCI, intrathecal administration of the enzyme improved locomotion and bladder control compared with what was seen in rats that received enzymatic protein control. ${ }^{7}$

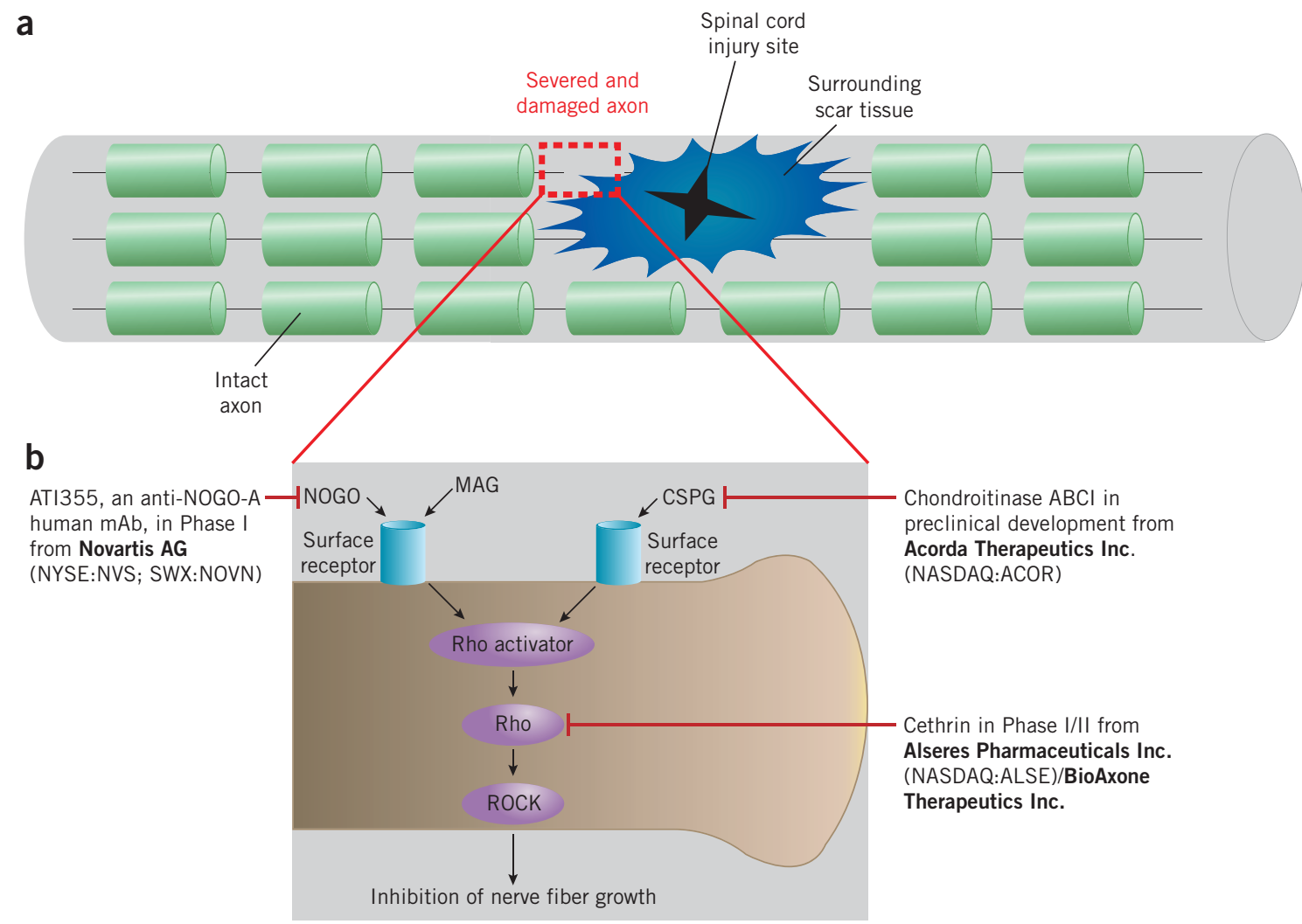

Figure 1. Blocking growth inhibition in spinal cord injury. A paper published in The Journal of Neuroscience by researchers at University of Texas Southwestern Medical School suggests that the marketed mood stabilizer lithium may have the capacity to regenerate damaged neurons in spinal cord injury (SCl). If so, the drug would join a growing class of compounds in preclinical and clinical development that target growth inhibitory pathways in SCl.

[a] In SCl, the sudden displacement of the bones and ligaments of the spinal column causes local injury to spinal cord tissue. A cascade of secondary cellular events then transform the initial injury site into a larger surrounding site characterized by scar tissue (the glial scar). The severed and damaged axons of neurons bordering the injury site are prevented from regrowth by multiple inhibitors in the glial scar.

[b] Growth inhibitors in the microenvironment of damaged axons include chondroitin sulfate proteoglycan (CSPG), myelin-associated glycoprotein (MAG) and the protein reticulon 4 (NOGO; NOGO-A). Each of these ligands and others can bind to receptors on the surface of neurons, triggering an intracellular pathway that ultimately leads to irreversible blockage of nerve fiber growth. The proteins Rho and Rhoassociated, coiled-coil containing protein kinase (ROCK) play a central role in this pathway.

Some companies in the SCI space have sought to develop compounds that block activation of the growth inhibitory pathway, either by preventing ligand-receptor interactions on the neuron's surface or by inhibiting downstream activation of Rho. 


\section{TARGETS \& MECHANISMS}

Table 1. Spinal cord injury pipeline. Treatments in development for spinal cord injury include cell-based therapeutics as well as compounds targeting neuronal growth inhibitory pathways. Below is a selected list of spinal cord injury (SCl) treatments in development.

\begin{tabular}{|c|c|c|c|}
\hline Company & Product & Description & Status \\
\hline Acorda Therapeutics Inc. (NASDAQ:ACOR) & Fampridine-SR & Sustained-release formulation of 4-aminopyridine & Phase III ${ }^{\mathrm{A}}$ \\
\hline $\begin{array}{l}\text { Alseres Pharmaceuticals Inc. (NASDAQ: } \\
\text { ALSE)/BioAxone Therapeutics Inc. }\end{array}$ & Cethrin & $\begin{array}{l}\text { Recombinant C3 exoenzyme protein, a Rho guanosine } \\
\text { triphosphatase antagonist, mixed with a fibrin sealant }\end{array}$ & Phase I/II \\
\hline Novartis AG (NYSE:NVS; SWX:NOVN) & ATI355 & Anti-reticulon 4 (NOGO-A) mAb & Phase I \\
\hline Aastrom Biosciences Inc. (NASDAQ:ASTM) & Neural repair cells (NRCs) & NRCs based on Tissue Repair Cell (TRC) technology & Preclinical \\
\hline Acorda & Chondroitinase $\mathrm{ABCI}$ & Bacterial enzyme that breaks down chondroitin sulfate proteoglycans & Preclinical \\
\hline $\begin{array}{l}\text { BrainStorm Cell Therapeutics Inc. } \\
\text { (OTCBB:BCLI) }\end{array}$ & $\begin{array}{l}\text { Neurotrophic factor cells } \\
\text { (NFCs) }\end{array}$ & $\begin{array}{l}\text { NFCs generated from adult human bone marrow-derived stem } \\
\text { cells }\end{array}$ & Preclinical \\
\hline Geron Corp. (NASDAQ:GERN) & GRNOPC1 & Human embryonic stem cell-derived oligodendrocytes & Preclinical \\
\hline Neuralstem Inc. (AMEX:CUR) & Human neural stem cells & Human neural stem cells (hNSCs) & Preclinical \\
\hline Sangamo BioSciences Inc. (NASDAQ:SGMO) & $\begin{array}{l}\text { Zinc finger DNA-binding } \\
\text { protein transcription factor } \\
(\text { ZFP TF) VEGF-A }\end{array}$ & ZFP TF upregulating the expression of a gene encoding VEGF-A & Preclinical \\
\hline StemCells Inc. (NASDAQ:STEM) & $\begin{array}{l}\text { Human CNS stem cells } \\
\text { (HuCNS-SC) }\end{array}$ & Neural stem cells & Preclinical \\
\hline Sygnis Pharma AG (Xetra:LIO) & AX200 & G-CSF & Preclinical \\
\hline
\end{tabular}

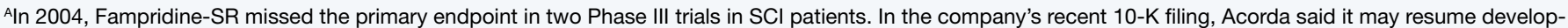
ment in $\mathrm{SCl}$ after it completes further development of the compound for multiple sclerosis (MS).

Work by a University of Hong Kong group has shown that lithium plus chondroitinase enhanced the axonal regeneration compared with the effects of chondroitinase alone in a rat model of SCI. ${ }^{8}$

Acorda's Fampridine-SR-an oral sustained-release 4-aminopyridine $\mathrm{K}$ channel blocker-failed to lower spasticity in two separate Phase III trials to treat chronic SCI, but it significantly improved bowel, bladder and sexual function. The compound has shown efficacy in an ongoing Phase III trial to treat multiple sclerosis (MS).

Curtis Tyree, director of scientific affairs at TorreyPines Therapeutics Inc., said GSK $3 \beta$ inhibition could play a part in strategies that treat muscle spasticity and rigidity following SCI. "For example, combining a GSK3 $\beta$ inhibitor with baclofen-standard of care for spasticity associated with spinal cord injury-could lead to additive improvements over baclofen treatment alone," he said. "Toward that end, I would like to see additional work on GSK $3 \beta$ combination therapies in rodent models of spinal ischemic spasticity and rigidity."

Intrathecal baclofen is a generic muscle relaxant marketed to treat spasticity in patients with spinal cord injury and MS.

TorreyPines' Tezampanel (NGX424), an $\alpha$-amino-3-hydroxy-5methyl-4-isoxazolepropionic acid glutamate antagonist, improved peripheral muscle function in a rat model of ischemic paraplegia. ${ }^{9}$ Tyree told SciBX the company is now discussing whether to run a Phase I trial in spasticity associated with SCI.

\section{Box 1. Inhibiting GSK3 in cancer.}

Other recently published preclinical research suggests glycogen synthase kinase $3 \beta$ (GSK3 $\beta$ ) inhibition might also have value in cancer. Last month, Michael Cleary and colleagues at Stanford University School of Medicine reported in Nature that inhibiting GSK3 $\beta$ helped treat a specific subtype of leukemia defined by mutations in the MLL proto-oncogene. ${ }^{10}$

GSK3 $\beta$ inhibition with SB216763 induced cell-cycle arrest in cultured $M L L$ leukemia cells, whereas non-MLL leukemia cells were minimally affected. Moreover, in mice transplanted with MLL-AF4 leukemia cells, chow laced with lithium carbonate increased survival compared with that of transplanted mice that ate regular chow.
Like compound SB415286 used in the $\mathrm{SCl}$ study, ${ }^{1} \mathrm{SB} 216763$ is also a small molecule competitive inhibitor of GSK3 $\beta$ that was originally developed by SmithKline Beecham, now part of GlaxoSmithKline plc. ${ }^{6}$

"Leukemias with $M L L$ genetic aberrations are generally associated with poor prognosis and treated with more aggressive chemotherapy regimens. Targeting GSK3 $\beta$ may provide another approach that could be employed in conjunction with standard chemotherapy," said Cleary, who is professor of pathology and pediatrics at Stanford.

Spiro Rombotis, president and CEO of Cyclacel Pharmaceuticals Inc., told $S c i B X$ that the findings are quite surprising, because previous work has sug- gested that inhibiting the enzyme would increase cancer cell proliferation. Indeed, he said, Cyclacel originally developed its GSK3 $\beta$ inhibitors in diabetes with the goal of avoiding potential cross-reactivity that could lead to upregulation of certain cancer pathways.

Although Cyclacel has focused its efforts in cancer on sapacitabine, Rombotis said the Nature findings raise the possibility of repurposing the company's GSK3 $\beta$ inhibitors to $M L L$ cancer.

Cleary told SciBX that his lab's initial goal "is to develop and test more potent GSK3 $\beta$ inhibitors in preclinical models of MLL leukemia, either alone or in combination with standard chemotherapy." 


\section{TARGETS \& MECHANISMS}

Li told SciBX that UT Southwestern is considering filing for IP protection to cover the GSK3 $\beta$ inhibition strategy in SCI.

REFERENCES

1. Dill, J. et al. J. Neurosci.; published online Sept. 2, 2008; doi:10.1523/JNEUROSCl.1178-08.2008

Contact: Shuxin Li, University of Texas Southwestern Medical Center, Dallas, Texas e-mail: shuxin.li@utsouthwestern.edu

2. Mueller, B. et al. Nat. Rev. Drug Discov. 4, 387-398 (2005)

3. Silver, J. \& Miller, J. Nat. Rev. Neurosci. 5, 146-156 (2004)

4. Yoshimura, T. et al. Cell 120, 137-149 (2005)

5. Zhou, F. et al. Neuron 42, 897-912 (2004)

6. Coghlan, M. et al. Chem. Biol. 7, 793-803 (2000)

7. Caggiano, A. et al. J. Neurotrauma 22, 226-239 (2005)

8. Yick, L. et al. J. Neurotrauma 21, 932-943 (2004)

9. Hefferan, M. et al. J. Neurosci. 27, 11179-11191 (2007)
10. Wang, Z. et al. Nature; published online Sept. 17, 2008; doi:10.1038/nature07284

Contact: Michael Cleary, Stanford University School of Medicine, Stanford, Calif.

e-mail: mcleary@stanford.edu

COMPANIES AND INSTUTIOTIONS MENTIONED

Acorda Therapeutics Inc. (NASDAQ:ACOR), Hawthorne, N.Y. Alseres Pharmaceuticals Inc. (NASDAQ:ALSE), Hopkinton, Mass. BioAxone Therapeutics Inc., Montreal, Quebec, Canada Cyclacel Pharmaceuticals Inc. (NASDAQ:CYCC), Berkeley Heights, N.J. GlaxoSmithKline plc (LSE:GSK; NYSE:GSK), London, U.K.

Stanford University School of Medicine, Stanford, Calif.

TorreyPines Therapeutics Inc., La Jolla, Calif.

University of Hong Kong, Hong Kong, China

University of North Carolina School of Medicine, Chapel Hill, N.C. University of Texas Southwestern Medical Center, Dallas, Texas 